\title{
Third order nonlinear optical constants of $L$-alaninium oxalate
}

\author{
K.J. Arun, ${ }^{* 1,2}$ S. Jayalekshmy ${ }^{2}$ \\ ${ }^{1}$ Department of Physics, Sree Kerala Varma College, Thrissur-680011, Kerala, India \\ ${ }^{2}$ DREAM Lab, Department of Physics, Cochin University of Science \& Technology, Kochi, Kerala, India.
}

Received September 13, 2015; accepted September 24, 2015; published September 30, 2015

\begin{abstract}
A charge transfer complex crystal L-alaninium oxalate (LAO) is grown by slow evaporation at ambient temperature using an equimolar solution of L-alanine and oxalic acid as precursors. The third order nonlinear optical constants of the thin cleaved LAO crystal is measured by a single beam $\mathrm{Z}$ scan technique with and without an aperture in the far field. The crystal shows reverse saturable absorption and negative nonlinear refraction under our experimental conditions Nonlinear optical constants are calculated from the experimental data.
\end{abstract}

Photonic sensors (including eye) have threshold intensity to electromagnetic radiation above which they can be damaged [1]. By using suitable materials as optical limiters, the dynamical range of the sensor can be extended to function optimally at higher input intensities. For most protective applications a rapid sensor response is desired with a large saturation threshold, providing a greater safety margin [2]. Various nonlinear optical effects can be employed for designing an optical limiter.

Nonlinear optical (NLO) materials with a large intensity dependent refractive index and absorption coefficients are useful for optical device applications. These parameters determine whether an intense laser beam will undergo self-focusing or self-defocusing as it propagates in the material medium [3].

Crystals of amino acids and their salts are bio organic NLO materials with promising applications in lasers and non-linear optics [4]. L-alaninium oxalate (LAO), which is the charge transfer complex of L-alanine and oxalic acid, is an interesting nonlinear optical material [5]-[6]. In the present work, good quality single crystals of LAO are grown using a simple and cost effective technique of solution growth and third order nonlinear optical properties are investigated using the $\mathrm{Z}$ scan technique.

L-alaninium oxalate is synthesized from an equimolar solution of L-alanine and L-oxalic acid by evaporation preventing decomposition. Seed crystals are grown to big crystals by slow evaporation to a size of about $40 \times 15 \times 8 \mathrm{~mm}^{3}$ with good transparency in a time of about three weeks as shown in Fig. 1. From the powder XRD

\footnotetext{
*E-mail: drarunkj@gmail.com
}

shown in Fig. 2, it is found that L-alaninium oxalate belongs to the orthorhombic system with $\mathrm{a}=5.6302 \AA$, $\mathrm{b}=7.235 \AA$ and $\mathrm{c}=19.5973 \AA$, the space group being the no centrosymmetric $\mathrm{P} 22_{1} 2_{1} 2_{1}$ and has four molecules in the unit cell with a volume of $803.146 \AA^{3}$. The close agreement with the observed, calculated and reported values [4] confirms the identity of the grown crystal. From the single crystal XRD analysis it is inferred that the amino group of the L-alaninium cation forms three $\mathrm{N}-$ $\mathrm{H}$... O hydrogen bonds with the oxygen of semi oxalate group and a symmetry-related alanine cation. There also exist two $\mathrm{O}-\mathrm{H}$... O hydrogen bonds. The alaninium and semi-oxalate ions form alternate columns leading to a layered arrangement parallel to the a.c. plane and each such layer is interconnected to the other through $\mathrm{N}-\mathrm{H}$... O hydrogen bonds.

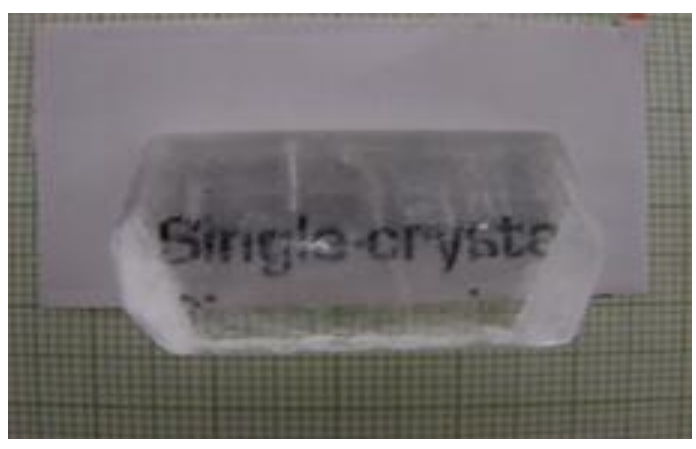

Fig.1. Grown single crystal.

The chemical composition of the grown crystal determined using CHN elemental analysis reveals that it contains $33.18 \%$ carbon $(33.51 \%), 4.96 \%$ hydrogen $(5.02 \%), 7.79 \%$ nitrogen $(7.82 \%)$ where the figures in the bracket represent a theoretical composition. Thus the molecular formula of the compound $\mathrm{L}$ alaninium oxalate is established as $\mathrm{C}_{5} \mathrm{H}_{9} \mathrm{NO}_{6}$ and it does not contain any water of crystallization in its structure. The UV/Vis/NIR absorption spectrum in Figure 3shows that LAO crystal has a wide transparency window ranging from $318 \mathrm{~nm}$ to $1524 \mathrm{~nm}$, suggesting its suitability for second and third harmonic generations of the $1064 \mathrm{~nm}$ radiation and other applications in the blue-violet region. The low absorption 
in the visible and near infra-red region and considerable absorption in the deep UV and far IR region enable its application as a suitable material for the construction of

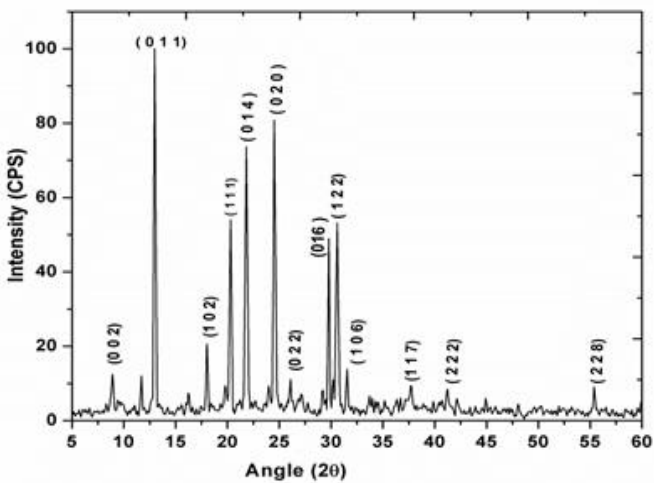

poultry roofs and walls and for coating eyeglasses as antireflection and thermal control coatings.

Fig. 2. Powder XRD spectrum.

The FT IR spectrum of the LAO crystal is recorded using an AVTAR 370 system with a resolution of $4 \mathrm{~cm}^{-1}$ in the range of $400 \div 4000 \mathrm{~cm}^{-1}$, being shown in Fig. 4. The shoulder band at $2902 \mathrm{~cm}^{-1}$ in the IR spectrum corresponds to $\mathrm{NH}_{3}{ }^{+}$symmetric stretch mode. The medium shoulder at $3200 \mathrm{~cm}^{-1}$ in the Raman spectrum shows $\mathrm{NH}_{3}{ }^{+}$asymmetric stretching mode. The positions of these bands clearly indicate the presence of an $\mathrm{NH}_{3}{ }^{+}$group in the crystal. The $\mathrm{NH}_{3}{ }^{+}$asymmetric and symmetric bending occur in the region $1625 \div 1560 \mathrm{~cm}^{-1}$ and $1550 \div 1500 \mathrm{~cm}^{-1}$, respectively. The presence of $\mathrm{NH}_{3}{ }^{+}$ion in the crystal confirms the protonation of the amino acid group leading to the formation of L-alaninium oxalate molecule.

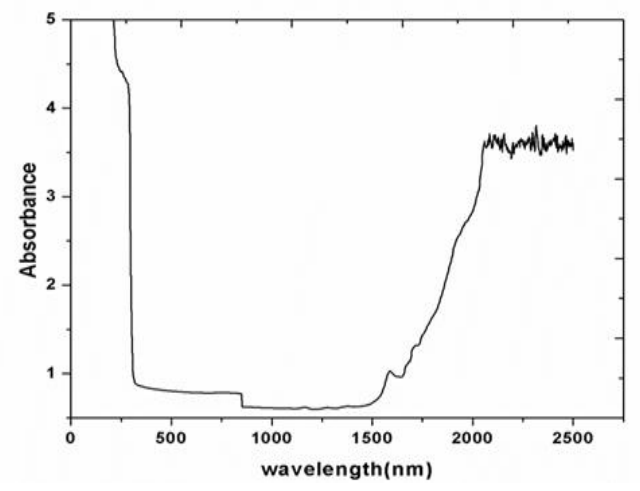

Fig.3. UV/Vis/VIR spectrum of the crystal.

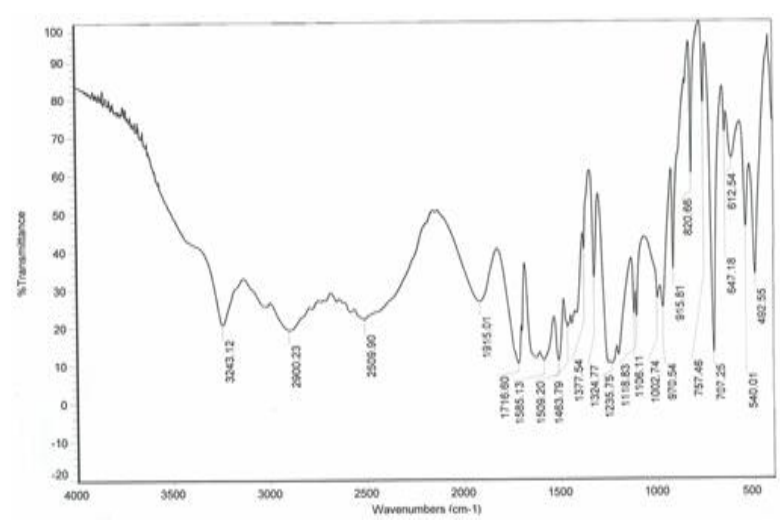

Fig. 4. FTIR spectrum.

Nonlinear optical characterization of the crystal is carried out using a well-known, single-beam, $\mathrm{Z}$ scan technique [7], which can accurately measure both the magnitude and sign of the nonlinear refractive index $n_{2}$. The measurements are carried out with the experimental setup shown in Fig. 5. The laser beam is provided by the output of a pulsed Nd: Yttrium Aluminum Garnet (YAG) laser, with a pulse width of $7 \mathrm{~ns}$ and a repetition rate of $10 \mathrm{~Hz}$. The thin cut crystal sample is placed on a translation stage controlled by a computer that moves the sample along the $z$-axis with respect to the focal point of a $50 \mathrm{~mm}$ convex lens. The beam waist radius in the focal plane is about $25 \mu \mathrm{m}$. The transmittance of the samples is measured with and without an aperture in the far-field. The laser pulses adjusted by an attenuator are separated into two beams by using a splitter. The energy of the two beams is simultaneously measured using two energy detectors, Rjp-735 and energy probes linked to energy ratio meter, $\mathrm{Rj}-7620$. A personal computer is used to collect and process the data coming from energy meters.

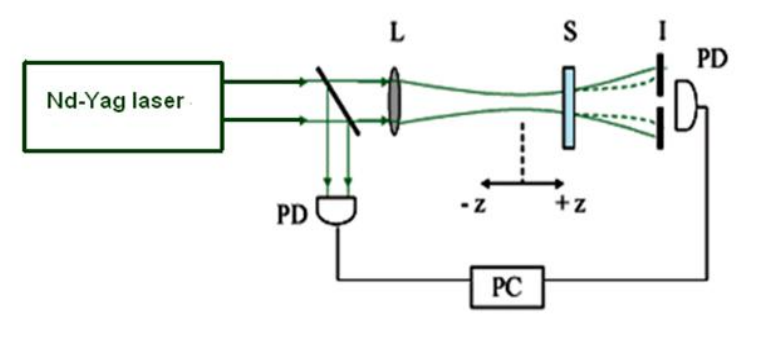

Fig.5. Z scan experimental set up.

The result of the open aperture $\mathrm{Z}$ scan measurement of the crystal is shown in Fig. 6(a). The open aperture (OA) curve demonstrates a nonlinear absorption and the characteristic pattern of the curve shows that the nonlinear absorption is reverse saturable absorption (RSA). For $532 \mathrm{~nm}$ resonant absorption, both excited state absorption 
and two-photon absorption (TPA) can be responsible for the observed NLO effects. The RSA coefficient $\beta$ can be obtained from the fitting performed on the experimental data of the OA measurement with Eqs. (1)-(2) [8], where $\alpha$ and $\beta$ are the linear and effective third order NLO absorption coefficients, respectively, $\tau$ is the time, $I(z)$ is the irradiance and $L$ is the optical path length. Since we are getting an excellent fit of the experimental data with Eqs. (1) and (2) corresponding to two photon absorption, it can be concluded that the two photon absorption is the mechanism responsible for the observed NLO effect.

$$
\begin{gathered}
T(z)=\frac{1}{Q(z) \sqrt{\pi}} \int_{-\infty}^{+\infty} \ln [1+Q(z)] e^{-\tau^{2}} \mathrm{~d} \tau, \\
Q(z)=\beta I(z) \frac{1-e^{-\alpha L}}{\alpha},
\end{gathered}
$$

where $\left(1-\mathrm{e}^{-\alpha L}\right) / \alpha$ is the effective path length $L_{e f f}$. The value of the nonlinear absorption coefficient $\beta$ is found to be equal to be $11.65 \mathrm{~cm} / \mathrm{GW}$.
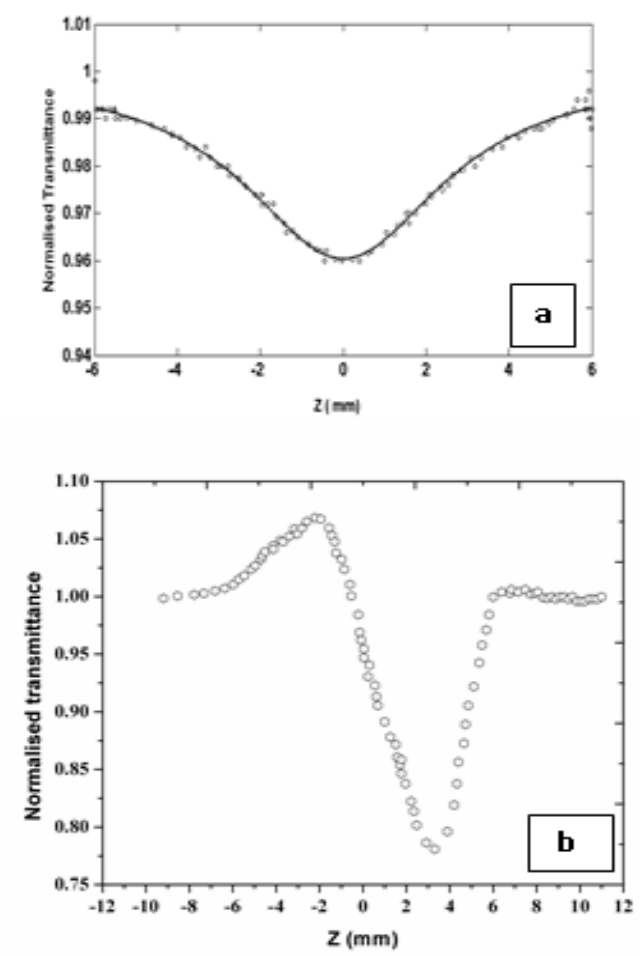

Fig. 6. Z scan curve (a) without and (b) with aperture.

The peak-to-valley configuration of the curve obtained under the closed aperture configuration in Fig. 6(b) suggests that the refractive index change is negative, exhibiting a self-defocusing effect. The nonlinear refraction index $n_{2}\left(\mathrm{~m}^{2} / \mathrm{W}\right)$ is obtained through the following equation:
$\Delta \Phi_{0}=k n_{2} I_{0} L_{e f f}$.

In the above equation the phase shift $\Delta \Phi_{0}$ is equal to $2 \pi n_{2} I_{0} L_{e f f} \lambda^{-1}$. The difference between the normalized transmittance at the peak and valley is related to $\Delta \Phi_{0}$ by the relation :

$\Delta \mathrm{T}_{\mathrm{P} \rightarrow \mathrm{V}}=0.406 \cdot(1-\mathrm{S})^{0.25} \Delta \Phi_{0}$.

The value of $n_{2}$ is calculated to be equal to $-3.984 \times 10^{-13}$ $\mathrm{cm}^{2} / \mathrm{W}$. The molecular hyperpolarizability, $\gamma$, is obtained to be $10.424 \times 10^{-24}$ esu with the equation below [9]:

$\gamma=40 \pi n_{2} / \mathrm{c} n_{0}$,

where $\mathrm{n}_{0}$ is the linear refractive index which is estimated from the absorption spectrum of the crystal. The real and imaginary parts of the $\chi^{(3)}$ of the sample can also be calculated by the following equations [9]:

$\operatorname{Re} \chi^{(3)}(\mathrm{esu})=\left(\mathrm{c} n_{0}{ }^{2} / 120 \pi^{2}\right) n_{2}$,

$\operatorname{Im} \chi^{(3)}(\mathrm{esu})=\left(\mathrm{c}_{0}{ }^{2} \varepsilon_{0} \lambda / 2 \pi\right) \beta$.

The value of the real part of nonlinear susceptibility $\mathrm{Re}$ $\chi^{(3)}(\mathrm{esu})$ is $-2.586 \times 10^{-11}$ and imaginary part $\operatorname{Im} \chi^{(3)}(\mathrm{esu})$ is $0.6376 \times 10^{-11}$.

Introducing the coupling factor $\rho$, the ratio of imaginary part to real part of third-order nonlinear susceptibility,

$\rho=\operatorname{Im} \chi^{(3)} / \operatorname{Re} \chi^{(3)}$.

The value of $\rho$ in this case is found to be equal to 0.24 . The observed value of the coupling factor is seen to be less than $1 / 3$, which indicates that the nonlinearity is electronic in origin. The third order nonlinear optical properties of the L-alaninium oxalate crystal are investigated by the $\mathrm{Z}$ scan technique. The crystal shows reverse saturable absorption and negative nonlinear refraction under our experimental conditions. The reason for the large value of RSA coefficient may be due to the protonation of amino group leading to the formation of a charge transfer complex.

\section{References}

[1] L.W. Tutt, T.F. Boggess, Prog. Quant. Electron. 17, 299 (1993).

[2] P.N.Prasad, D.J. Williams, Introduction to Nonlinear Optical Effects in Molecules and Polymers ( New York, Wiley1991).

[3] M.Subhandinini et al., Acta Crystallogr. E 57, 633 (2001).

[4] S. Dhanushkodi, K. Vasantha, Cryst.Res.Technol. 39, 259 (2004).

[5] K.J. Arun, S. Jayalekshmi, Optoelectron. Adv. Mater. 2, 805 (2008)

[6] M. Sheik-Bahae et al., IEEE J. Quantum Electron. 26, 760 (1990).

[7] M. Sheik-Bahae, A.A. Said et al., Opt. Lett. 14, 955 (1989).

[8] S.F. Wang, W.T. Huang, T.Q. Zhang, Appl. Phys. Lett. 75, 1845 (1999)

[9] K.J. Arun, S.Jayalekshmi, Optoelectron. Lett. 7(2), 136 (2011). 